\title{
Bi-monthly hepatic arterial infusion chemotherapy as a novel strategy for advanced hepatocellular carcinoma in decompensated cirrhotic patients
}

\author{
Kei Moriya', Tadashi Namisaki', Shinya Sato', Masanori Furukawa', Akitoshi Douhara', Hideto Kawaratani', \\ Kosuke Kaji', Naotaka Shimozato', Yasuhiko Sawada', Soichiro Saikawa', Hiroaki Takaya', Koh Kitagawa', \\ Takemi Akahane', Akira Mitoro', Junichi Yamao², and Hitoshi Yoshiji ${ }^{1}$ \\ 'Division of Gastroenterology and Hepatology, Third Department of Internal Medicine, Nara Medical University, Kashihara; ${ }^{2}$ Depart- \\ ment of Endoscopy, Nara Medical University, Kashihara, Japan
}

Background/Aims: We previously reported the comparable efficacy of bi-monthly hepatic arterial infusion chemotherapy (B-HAIC) to that of sorafenib chemotherapy for the treatment of advanced hepatocellular carcinoma (aHCC) in patients with compensated cirrhosis. In this study, we demonstrate the efficacy of B-HAIC in patients with decompensated cirrhosis.

Methods: Forty-five patients with aHCC refractory to transcatheter arterial chemo-embolization (TACE) were treated with B-HAIC and were divided into two groups according to hepatic functional reserve (Child-Pugh grade). Overall survival period, treatment response, and adverse events in each group were analyzed.

Results: Efficacy and disease control rates in the Child-Pugh B group ( $n=24 ; 21 \%$ and $71 \%$, respectively) were not significantly impaired compared the Child-Pugh A group ( $n=21 ; 38 \%$ and $67 \%$, respectively). Median survival time and survival rate at 12 months in the Child-Pugh B group were 422 days and $58.3 \%$, respectively, whereas those in the ChildPugh A group were 567 days and $70.8 \%$, respectively. Importantly, the hepatic functional reserve of patients did not worsen in either group during the treatment period. Furthermore, the occurrence rate of adverse events leading to discontinuation of anti-tumor treatment was not significantly increased in the Child-Pugh B group.

Conclusions: Given the preservation of hepatic functional reserve afforded by B-HAIC chemotherapy in patients with decompensated cirrhosis, B-HAIC might be an acceptable alternative strategy for aHCC patients who do not respond to TACE. (Clin Mol Hepatol 2019;25:381-389)

Keywords: Carcinoma, Hepatocellular; Liver cirrhosis; Cisplatin; Drug therapy; Decompensated cirrhosis

\footnotetext{
Study Highlights

Efficacy of bi-monthly hepatic arterial infusion chemotherapy (B-HAIC) on advanced hepatocellular carcinoma in patients with compensated cirrhosis had already been reported as comparable to that of sorafenib chemotherapy. Efficacy and disease control rates of B-HAIC in the decompensated cirrhotic patients were not impaired to those of compensated cirrhotic patients and the hepatic functional reserve of patients did not worsen even in the patients with relatively poor hepatic functional reserve during the treatment period. Then, B-HAIC may be acceptable as an alternative strategy for patients with advanced hepatocellular carcinoma who do not respond to transcatheter arterial chemoembolization.
}

\section{Abbreviations:}

aHCC, advanced hepatocellular carcinoma; B-HAIC, bi-monthly hepatic arterial infusion chemotherapy; CT, computed tomography; HAIC, hepatic arterial infusion chemotherapy; HCC, hepatocellular carcinoma; MRI, magnetic resonance imaging; TACE, transcatheter arterial chemoembolization

\section{Corresponding author : Kei Moriya}

Division of Gastroenterology and Hepatology, Third Department of Internal Medicine, Nara Medical University, 840 Shijo-cho, Kashihara, Nara 634-8522, Japan

Tel: +81-744-22-3051, Fax: +81-744-24-7122

E-mail:moriyak@naramed-u.ac.jp

https://orcid.org/0000-0002-7500-6728 


\section{INTRODUCTION}

Hepatocellular carcinoma (HCC) has an incidence of 0.84 million per year and is the sixth most common neoplasm in the world.' The total number of annual deaths due to HCC is 0.78 million, the second highest of all cancer-related deaths.' Based on these data, the rate of HCC-related deaths to the incidence of this cancer is extremely high (about 93\%), justifying the fact that HCC has a very poor prognosis.

In fact, approximately $70-90 \%$ of HCC cases occur in patients with chronic liver diseases, such as chronic hepatitis and liver cirrhosis. The conventional etiologies of chronic liver disease are human hepatitis B virus infection, human hepatitis $C$ virus infection, and excess alcohol consumption. Currently, metabolic syndrome and excessive obesity are also known to cause not only fatty liver, but also steatohepatitis that may progress to liver cirrhosis.

Liver cancer is generally diagnosed in an advanced clinical stage because this disease tends to be clinically silent during the early stages. Moreover, clinicians should consider the hepatic functional reserve level of each patient during treatment selection.

The anti-tumor agents, sorafenib and lenvatinib, are shown to be useful in the treatment of advanced $\mathrm{HCC}(\mathrm{aHCC})^{2,3}$ and are the only two established first-line chemotherapeutic agents for aHCC. However, both these drugs affect the patient's hepatic functional reserve because of their effects on the metabolic pathway that functions throughout the liver. ${ }^{4,5}$ Therefore, the decision to use these agents should be based on prior appropriate assessments of the hepatic functional reserve. Most aHCC patients have limited normal hepatic function and concomitant disease, and their prognosis partially depends on their hepatic functional reserve. That is why these molecular targeted drugs are permitted only for patients with compensated cirrhosis, not for those with decompensated cirrhosis. ${ }^{6,7}$ By the way, hepatic arterial infusion chemotherapy (HAIC) allows the repetitive delivery of high intrahepatic drug concentrations without the need of synchronous embolization of the hepatic vasculature and results in acceptable toxicity levels including hepatic damages.

In this study, we hypothesized that a bi-monthly HAIC (B-HAIC) protocol would be useful for treating patients with decompensated cirrhosis who have aHCC. Thereafter, we compared the outcomes of this approach with previously reported outcomes of arterial infusion chemotherapy using a reservoir. ${ }^{8-14}$

\section{PATIENTS AND METHODS}

\section{Patients}

To evaluate the efficacy and safety of B-HAIC for patients with aHCC and cirrhosis, the records of 96 chemo-naive patients refractory to transcatheter arterial chemoembolization (TACE) or those who demonstrated distinct extrahepatic metastatic lesions were reviewed. The eligible patients were admitted to the Nara Medical University Hospital from January 2009 to December 2014 and were enrolled in this retrospective study. Fifty of the 96 patients were then excluded because they received prior treatment with sorafenib following treatment failure with TACE. Forty-five of the 46 patients treated with B-HAIC were finally enrolled and divided into two different groups as per the grade of their hepatic functional reserve. One patient who died because of acute disease progression within 4 weeks of treatment initiation was excluded. For the classification of HCC, we used the TNM staging system adopted by the Union for International Cancer Control in this study.

\section{Definition of TACE failure}

The preceding TACE sessions were performed using an emulsion containing anticancer agents and lipiodol followed by the application of gelatin sponge particles. The TACE refractory patients were diagnosed as per the guidelines of the Japan Society of Hepatology and the Liver Cancer Study Group of Japan. ${ }^{15,16}$ For intrahepatic lesions, TACE failure was defined as $\geq 2$ consecutive ineffective responses of treated tumors (viable lesions $>50 \%$ ) or $\geq 2$ consecutive progressive increases in the total tumor counts, in spite of a prior change in the choice of chemotherapeutic agent or re-analysis of the feeding artery. Ineffective responses were determined by using computed tomography (CT) or magnetic resonance imaging (MRI) 30-90 days after an adequately performed selective TACE procedure. Other TACE failure criteria included the continuous elevation of tumor marker levels immediately after TACE and the appearance of vascular invasion and extrahepatic spread. HCC diagnosis was established on the basis of characteristic radiological findings with increases in the serum alpha-fetoprotein (including its L3 population) and/or des-gamma carboxyprothrombin levels. ${ }^{17,18}$

\section{B-HAIC treatments}

For B-HAIC using cisplatin, intra-arterial cisplatin at $65 \mathrm{mg} / \mathrm{m}^{2}$ 
was basically administered over 30 minutes via a catheter inserted into the right hepatic artery or the left hepatic artery every 8 weeks, for up to six courses, until disease progression, or until unacceptable adverse events occurred. Reduced dose of cisplatin $\left(45 \mathrm{mg} / \mathrm{m}^{2}\right)$ was adopted in the two cases each in both the ChildPugh class A and class B disease through the course of their treatments. For the purpose of preventing renal toxicity of this drug, an infusion of $\geq 3,000 \mathrm{~mL}$ of extracellular fluid was administered on the day of B-HAIC, and an infusion of $\geq 1,000 \mathrm{~mL}$ was administered on the next day. Diuretics were generally administered for several days in cases of inadequate urine output. Patients who exhibited desirable response to the six courses of B-HAIC were then sequentially treated with an implanted 5 -fluorouracil reservoir. This treatment was continued until it was tolerated by the patient without disease progression or the occurrence of serious adverse effects.

\section{Assessment and statistical analyses}

Every 4-8 weeks, dynamic enhanced CT or MRI was used to confirm the anti-tumor effects of the treatment as per the modified Response Evaluation Criteria in Solid Tumors. ${ }^{19}$ The overall survival was calculated as the period from treatment initiation to the day of the patient's death or the final day of confirmed survival. Time to progression was defined as the period from the first day of treatment to the day of confirmation of tumor progression, as per radiological image examination. Adverse events were de- fined as the case with the Common Terminology Criteria for Adverse Events version 4.0 grade 2 or the more severe one in this study. ${ }^{20}$ Chi-square test and Mann-Whitney $U$ test were used to compare the patient characteristics and anti-tumor effects between the Child-Pugh A group and the Child-Pugh B group. We calculated the overall survival using the Kaplan-Meier method and compared the between-group differences using the log-rank test. $P<0.05$ was considered statistically significant. JMP version 11.2 (SAS Institute Inc., Cary, NC, USA) software was used for all the statistical analyses.

\section{Ethical issues}

Informed consent for each treatment was obtained from all the patients before treatment initiation. The Ethics Committee of the Nara Medical University Hospital approved this study (approval \#001490) that was conducted as per the ethical principles in the Japanese ethics guideline for epidemiological research. ${ }^{21}$

\section{RESULTS}

\section{Patient characteristics}

Of the 385 HCC patients in our hospital during the data collection period, only 96 patients with the following characteristics were included in the study: those with aHCC, those who were re-

Flow chart of the study patients

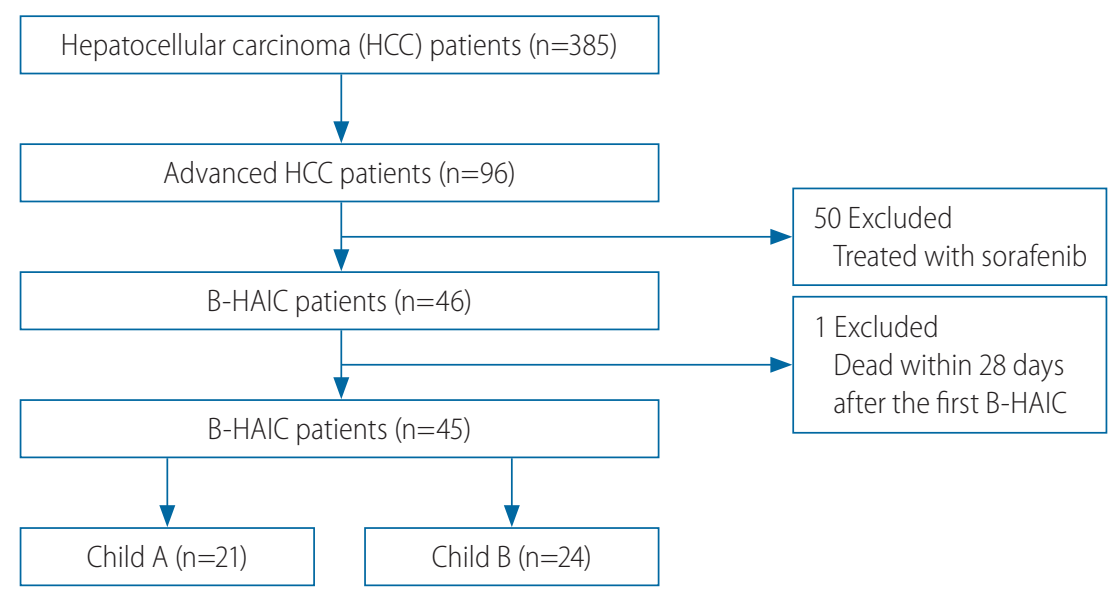

Figure 1. Patient flow chart. Three hundred eighty-five patients with HCC were initially included in analysis; of these, 96 had advanced HCC. Fifty of these 96 patients were excluded because they received treatment with sorafenib following treatment failure with TACE. Forty-five of the 46 patients treated with B-HAIC were enrolled and were divided into two groups based on hepatic functional reserve. B-HAIC, bi-monthly hepatic arterial infusion chemotherapy; TACE, transcatheter arterial chemoembolization. 
fractory to TACE, those who had undergone adequate radiological imaging assessments, and those who had undergone sufficient blood tests. Thereafter, we excluded 50 patients treated with sorafenib and another patient, as explained in the patients and methods section. We analyzed the records of 21 patients with compensated cirrhosis (Child-Pugh class A disease) and 24 with decompensated cirrhosis (Child-Pugh class B disease) (Fig. 1). The average interval between the B-HAIC cycles in the Child-Pugh class A group and the Child-Pugh class B group was $1.9 \pm 0.4$ and $1.8 \pm 0.6$ months (mean \pm standard deviation) respectively, and the average number of repeated cycles was $3.3 \pm 2.0$ and $3.0 \pm 1.2$ times, respectively. The total duration of the B-HAIC treatment averaged $6.2 \pm 3.7$ and $6.1 \pm 3.4$ months for the Child-Pugh class A and Child-Pugh class B groups, respectively. The profiles of the aHCC patients in both the groups are presented in Table 1. The patients in the Child-Pugh class A group $(n=21)$ and the ChildPugh class B group ( $n=24)$ with aHCC were comparable in terms of age, sex, and preceding treatment ratio. Patients in both the groups exhibited no extrahepatic metastasis (with one exception each) and were not significantly different in terms of the classifications of the number or the clinical stage of HCC. However, the population of patients with intravascular invasion was significantly larger in the Child-Pugh class A disease.

Table 1. Profiles of HCC patients with liver cirrhosis in this study ( $n=45$ )

\begin{tabular}{lccc}
\hline Hepatic functional reserve & Child $\mathbf{A}(\mathbf{n}=\mathbf{2 1})$ & Child B (n=24) & $\boldsymbol{P}$-value \\
\hline Age (years) & $69(44-88)$ & $56-82(72)$ & N.S. \\
Sex (male/female) & $16 / 5$ & $19 / 5$ & N.S. \\
\hline HCC numbers (1-3/4 and over) & $6 / 15$ & $5 / 19$ & N.S. \\
\hline Metastasis (yes/no) & $1 / 20$ & $1 / 23$ & N.S. \\
Intravascular invasion (with/without) & $10 / 11$ & $1 / 23$ & $<0.05$ \\
\hline HCC clinical stage (II/III/IV) & $3 / 13 / 5$ & $11 / 11 / 2$ & N.S. \\
\hline Tumor stage (T2/T3/T4) & $3 / 14 / 4$ & $9 / 13 / 2$ & N.S. \\
\hline AFP (ng/mL) & $948(2.6-406,875)$ & $46(3.2-109,267)$ & N.S. \\
\hline DCP (mAU/mL) & $405(10-268,747)$ & $85(8-15,459)$ & N.S. \\
Preceding medical treatments (yes/no) & $21 / 0$ & $24 / 0$ & N.S. \\
\hline
\end{tabular}

Values are presented as median (range) unless otherwise indicated.

Categorical variables were tested with Fisher's exact test and continuous variables with Welch's two sample $t$-test. HCC, hepatocellular carcinoma; N.S., not significant; AFP, alpha-fetoprotein; DCP, des-gamma-carboxy prothrombin.
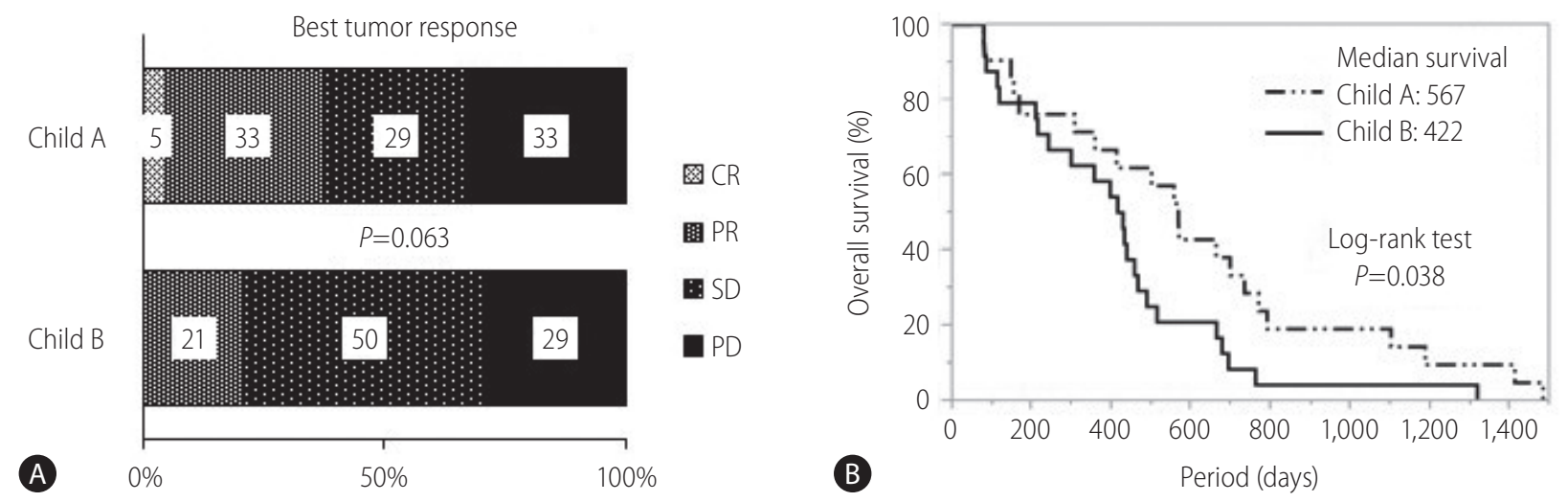

Figure 2. Chemotherapeutic effects of B-HAIC on advanced HCC. (A) Patients with Child-Pugh class A disease who were treated with B-HAIC were more likely to respond to treatment, while those with Child-Pugh class B disease who were treated with B-HAIC exhibited more stable disease rates. (B) Kaplan-Meier curves show the overall survival of patients with Child-Pugh class A disease (dashed line) and Child-Pugh class B disease (solid line) who were treated with B-HAIC. CR, complete response; PR, partial response; SD, stable disease; PD, progressive disease; B-HAIC, bi-monthly hepatic arterial infusion chemotherapy; HCC, hepatocellular carcinoma. 


\section{Efficacy and adverse events}

In this retrospective cohort study, the best tumor response and overall survival rate for each group are shown in Fig. 2. The disease control rate (Child-Pugh class A: $67 \%$, Child-Pugh class B: $71 \%$ ) was similar between the groups, while the efficacy rate in the Child-Pugh class A group was almost double of that in the Child-Pugh class B group ( $38 \%$ vs. $21 \%, P=0.063$ ). The calculated overall survival period of the Child-Pugh class $A$ group was significantly longer than that of the Child-Pugh class B group $(P=0.038)$. The median survival time and the survival rates at 6 , 12 , and 24 months were 567 days, 79.2\%, 70.8\%, and 33.3\%, respectively, in the Child-Pugh class $A$ group patients and 422 days, $79.2 \%, 58.3 \%$, and $8.3 \%$, respectively, in the ChildPugh class B group patients. It is noteworthy that the hepatic functional reserve in the patients of both groups did not change significantly during the treatment period $(P=0.346$ and $P=0.080$, respectively) (Fig. 3A). We observed no detrimental effects on the renal function in patients of either group $(P=0.554$ and $P=0.481$, respectively) (Fig. 3B). The main reason for treatment discontinuance in the Child-Pugh class A group patients was disease progression (19 out of 21 cases: $90.4 \%$ ); in contrast, $20 \%$ of those (5 out of 24 cases) in the Child-Pugh class B group discontinued treatment because of the following adverse events; two cases of hepatic artery obstruction, two cases of dysfunction of reservoir system due to mechanical trouble, and a case of rhabdomyolysis (Fig. 3C). However, no significant difference was observed between these two groups $(P=0.319)$. Patients in the Child-Pugh class A group exhibited a higher rate of additional chemotherapy, such as 5-fluorouracil-based regimens and/or sorafenib, after the end of the B-HAIC treatments as compared with the Child-Pugh class B group ( $P=0.010)$ (Fig. 3D)
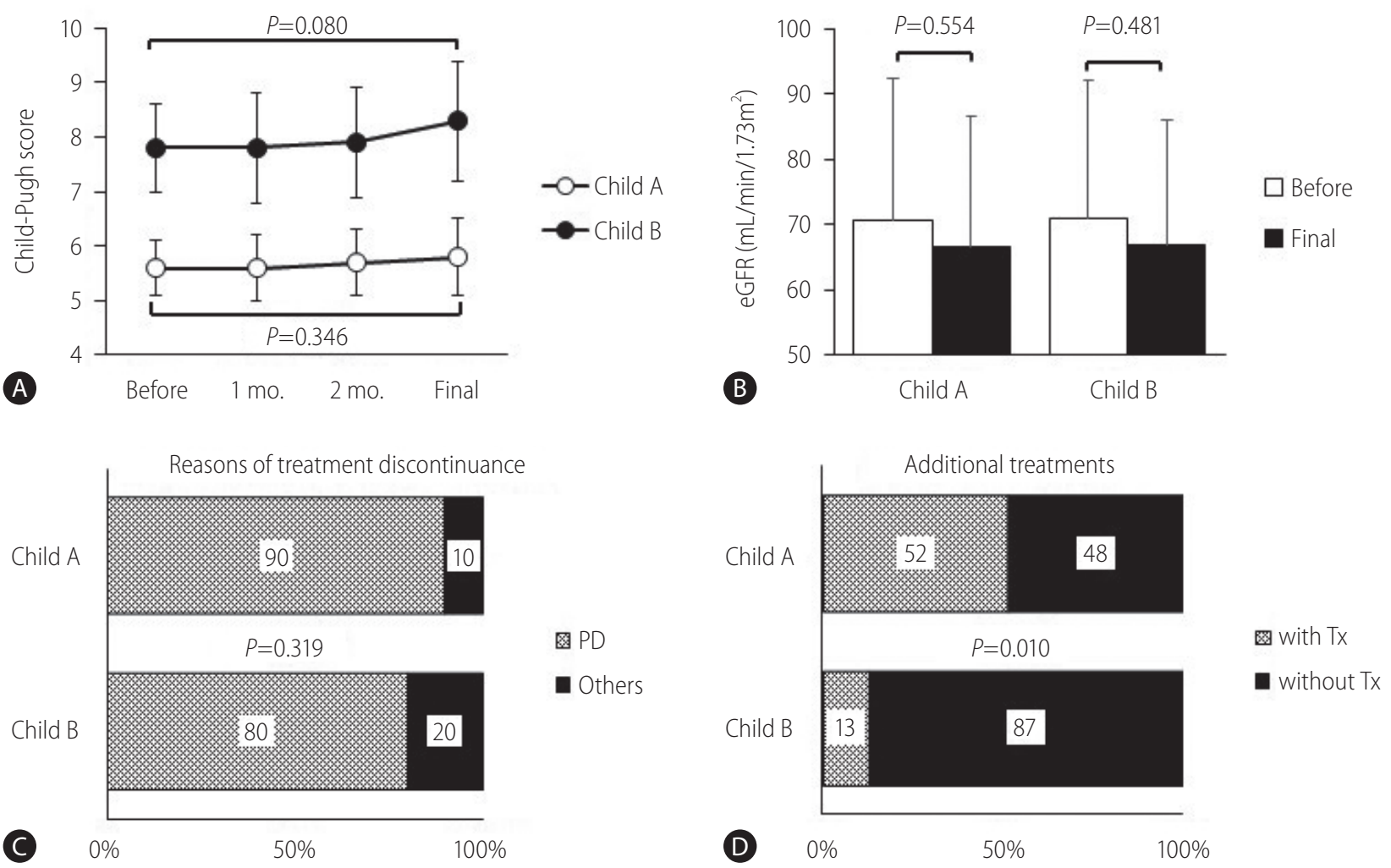

Figure 3. Adverse effects of B-HAIC in cases of advanced HCC. (A, B) Changes in Child-Pugh scores and estimated glomerular filtration rate (eGFR) before and after treatment in each group. Child-Pugh scores did not increase significantly during the B-HAIC treatment period in either group. (C) The majority of the patients had to discontinue B-HAIC due to disease progression despite hepatic functional reserve. (D) Patients with Child-Pugh class A disease who were treated with B-HAIC exhibited a significantly higher rate of additional chemotherapy than those with Child-Pugh class B disease. mo., month(s); PD, progressive disease; Tx, additional treatments; B-HAIC, bi-monthly hepatic arterial infusion chemotherapy; HCC, hepatocellular carcinoma. 


\section{DISCUSSION}

As per the current guidelines for the appropriate use of molecular-targeted drugs against HCC, these drugs should be prescribed only for patients with compensated cirrhosis and chronic hepatitis. ${ }^{6,7}$ In contrast, a B-HAIC regimen can be applied not only in patients with compensated cirrhosis, but also in those with decompensated cirrhosis classified as Child-Pugh class B disease.

In the present study, the average disease control rate in the patients with decompensated cirrhosis reached $70 \%$ and their median survival time and survival rate at 12 months were 422 days and $58.3 \%$, respectively (Fig. 2). The modest outcome in term of median survival period in decompensated cirrhotic patients as compared to that in the compensated patients was not attributable to their poor reactivity on B-HAIC but to the their own hepatic functional reserve level at the beginning of B-HAIC treatment. It is important to note that the hepatic functional reserves of these patients did not worsen during the treatment period as they did for those with compensated cirrhosis (Fig. 3A).

Currently, for the treatment of HCC in a specific patient, various options, such as surgical resection, radiofrequency ablation, and several types of chemotherapeutic agents are available. TACE was first established in Japan in 1978 and has been shown to be more effective than the best supportive care in randomized control studies and two meta-analyses. ${ }^{22-27}$ However, this chemotherapy regimen can cause loss of hepatic functional reserve. ${ }^{28-30}$ For cirrhotic patients whose life expectancy depends on their hepatic functional reserve, ${ }^{31,32}$ the maintenance of their own hepatic functional reserve at the highest possible level is crucial for effective HCC treatment.

Moreover, in cases of conventional HAIC, a reservoir device should be semi-permanently placed into the intrahepatic arterial space. In contrast, our B-HAIC does not require any transient device; however, a vascular puncture for catheter manipulation is generated for each infusion. ${ }^{33}$ In fact, conventional HAIC using a transient device is considered superior because the activity of the fine-powder formulation of cisplatin used in the puncture infusion method appears to have only modest anti-tumor activity and can be conducted only every 4-6 weeks. ${ }^{34-36}$ In contrast, the complication rates related to the use of a transient device (hepatic artery obstruction, pseudo arterial aneurysm formation, and port-related problems) are relatively high with the range between $2-56 \%$ (Table 2). Furthermore, some of these complications could negatively impact a patient's hepatic functional reserve. Thus, the person who is adminitered long-term repeated hepatic arterial chemotherapy infusions with a reservoir need to be well versed in the technique.

We hypothesized that B-HAIC may be better even for patients with relatively poor hepatic functional reserve because B-HAIC is simple, easy to manage, and is not frequently associated with

Table 2. Outcomes of 5-fluorouracil/cisplatin therapy on advanced HCC (repeated every 2-4 weeks)

\begin{tabular}{|c|c|c|c|c|c|c|c|c|c|c|c|}
\hline \multirow{2}{*}{ Study } & \multirow{2}{*}{$\begin{array}{l}\text { Adverse } \\
\text { events } \\
(\%)\end{array}$} & \multicolumn{3}{|c|}{ Cases* } & \multicolumn{3}{|c|}{$\mathrm{MST}^{\dagger}$ (months) } & \multicolumn{4}{|c|}{ Treatment response } \\
\hline & & Total & Child A & Child B & Total & Child A & Child B & CR & PR & SD & PD \\
\hline $\begin{array}{l}\text { Miyaki et al. } \\
\text { (2012) }\end{array}$ & 17 & 249 & 173 & 76 & 8.2 & 9.7 & 5.1 & \multicolumn{2}{|c|}{$\begin{array}{l}52 \text { (Child A) } \\
16 \text { (Child B) }\end{array}$} & $\begin{array}{l}63 \text { (Child A) } \\
20 \text { (Child B) }\end{array}$ & $\begin{array}{l}44 \text { (Child A) } \\
23 \text { (Child B) }\end{array}$ \\
\hline Oh et al. ${ }^{9}$ (2013) & 43 & 54 & 24 & 30 & 5.1 & 8.7 & 3.7 & \multicolumn{2}{|c|}{$\begin{array}{l}6(\text { Child A) } \\
6(\text { Child B) }\end{array}$} & $\begin{array}{l}9(\text { Child A) } \\
12 \text { (Child B) }\end{array}$ & $\begin{array}{l}6(\text { Child A) } \\
8 \text { (Child B) }\end{array}$ \\
\hline $\begin{array}{l}\text { Niizeki et al. } \\
\text { (2012) }\end{array}$ & 14 & 71 & 43 & 28 & 10.2 & $\mathrm{~N} / \mathrm{A}$ & $\mathrm{N} / \mathrm{A}$ & \multicolumn{2}{|c|}{$\begin{array}{l}18 \text { (Child A) } \\
7 \text { (Child B) }\end{array}$} & \multicolumn{2}{|c|}{$\begin{array}{l}25 \text { (Child A) } \\
21 \text { (Child B) }\end{array}$} \\
\hline Tsai et al. ${ }^{12}$ (2014) & 2 & 58 & 30 & 28 & 9.5 & N/A & N/A & N/A & $\mathrm{N} / \mathrm{A}$ & N/A & N/A \\
\hline $\begin{array}{l}\text { Terashima et al. } .^{13} \\
\text { (2014) }\end{array}$ & 19 & 27 & 9 & 18 & 7.6 & $\mathrm{~N} / \mathrm{A}$ & $\mathrm{N} / \mathrm{A}$ & \multicolumn{2}{|c|}{$\begin{array}{l}1 \text { (Child A) } \\
7 \text { (Child B) }\end{array}$} & $\begin{array}{l}5 \text { (Child A) } \\
4 \text { (Child B) }\end{array}$ & $\begin{array}{l}3 \text { (Child A) } \\
6 \text { (Child B) }\end{array}$ \\
\hline Song et al. ${ }^{8}$ (2015) & 56 & 50 & 45 & 5 & 7.1 & $\mathrm{~N} / \mathrm{A}$ & N/A & 1 & 11 & 33 & 5 \\
\hline Shao et al..$^{14}$ (2013) & 26 & 23 & 19 & 4 & 7.5 & 7.5 & 5.2 & $\begin{array}{l}6(C \\
0(C\end{array}$ & & $\begin{array}{l}7 \text { (Child A) } \\
2 \text { (Child B) }\end{array}$ & $\begin{array}{l}6(\text { Child A) } \\
1(\text { Child B) }\end{array}$ \\
\hline
\end{tabular}

HCC, hepatocellular carcinoma; MST, median survival time; CR, complete response; PR, partial response; SD, stable disease; PD, progress disease; N/A, not applicable.

*Number: 532 (total), 343 (Child A), and 189 (Child B).

${ }^{\dagger}$ Mean \pm standard deviation: $7.9 \pm 1.5$ (total), $8.6 \pm 0.9$ (Child A), and $4.7 \pm 0.7$ (Child B). 
Kei Moriya, et al.

complications. ${ }^{33}$ In the present study, we demonstrated that the hepatic functional reserve and renal function were not significantly decreased during the entire treatment period of B-HAIC in HCC patients. As a physical aspect, the non-invasiveness of B-HAIC might explain the satisfactory overall survival period; those patients also had an advantage compared with patients in whom a chemotherapy reservoir was placed.

For patients with compensated cirrhosis and HCC refractory to TACE, HAIC would have an efficacy similar to that of sorafenib, although the outcomes after either sorafenib or HAIC treatment in aHCC patients remain controversial. ${ }^{37-39}$ However, as mentioned above, patients with decompensated cirrhosis and aHCC are not considered good candidates for molecular-targeted therapy. ${ }^{6,7}$

In this retrospective cohort study, we showed the efficacy of BHAIC in patients with decompensated cirrhosis and aHCC. Although our study included only a small group of aHCC patients, the disease control rate and overall survival rate of patients who were classified as Child-Pugh class B and were treated with BHAIC were similar to those in patients treated with conventional HAIC with a reservoir. ${ }^{8-14}$ The patients with decompensated cirrhosis in this study were effectively treated with B-HAIC without any serious adverse events. From the viewpoint of not only maintaining hepatic functional reserve but also preventing the risk of cisplatin related adverse event such as hearing disorder, the sequential combination method of chemotherapy that comprises the initial administration of B-HAIC and the sequential use of conventional HAIC may lead to favorable outcomes.

In fact, our study population was not sufficiently large as those in other reports, ${ }^{9-12}$ because the B-HAIC was a novel strategy for advanced HCC and adopted only in our facility. And this was a retrospective study; therefore, we could not adjust for the prevalence of some clinical factors, such as age, sex, clinical stage of HCC, and sequential adjuvant chemotherapy. However, we regarded the hepatic functional reserve of cirrhotic patients as the most important factor in the treatment of their hepatic neoplasms. Therefore, we divided the patients in this study into two different groups as per their Child-Pugh classification in this clinical study.

In spite of certain limitations, to our knowledge, this is the first to demonstrate the efficacy of B-HAIC in patients with decompensated cirrhosis whose disease was classified as Child-Pugh class B. As shown in Table 2, several reports have demonstrated the usefulness of reservoir chemotherapy for fluorouracil-cisplatin regimens in patients with liver cirrhosis with Child-Pugh class $A$ and $B$ disease and aHCC. The reported median survival time in these report ranges from 5.1-10.2 months, ${ }^{8-14}$ and none of the survival times were higher than the survival period in patients treated with our B-HAIC method.

The median overall survival time in patients with decompensated cirrhosis in the B-HAIC group was almost 422 days and was distinctly comparable to that in patients who received conventional arterial infusion chemotherapy with a reservoir. B-HAIC is simple, easy to manage, and is widely available. It could be used even in patients with relatively poor hepatic functional reserve, such as those classified with Child-Pugh class B disease.

Thus, our results show that B-HAIC preserves the hepatic function even in patients with decompensated cirrhosis, suggesting that B-HAIC might be acceptable as an alternative strategy for aHCC patients who do not respond to TACE.

\section{Authors' contribution}

Concept and design: KM, JY, and HY. Clinical data collection: MF, HK, KK, NS, YS, SS, HT, KK, TA, and AM. Writing of article: KM. Data analysis: TN, SS, and AD. All the authors have seen and approved the final version of this manuscript.

\section{Acknowledgements}

The study was supported in part by a grant-in-aid from Zeria Pharmaceutical Co. Ltd. and Taisho Toyama Pharmaceutical Co. Ltd. and a grant-in-aid for research from Nara Medical University. Additionally, we thank Prof. Hiroshi Fukui for his kind and appropriate advice for completing this article.

\section{Funding support}

The study was supported in part by a grant-in-aid from Zeria Pharmaceutical Co. Ltd. and Taisho Toyama Pharmaceutical Co. Ltd. and a grant-in-aid for research from Nara Medical University.

\section{Conflicts of Interest}

The authors have no conflicts to disclose.

\section{REFERENCES}

1. World Health Organization (WHO). Cancer Today. WHO web site, $<$ http://gco.iarc.fr/today/fact-sheets-cancers $>$. Accessed 15 Jun 2019.

2. Llovet JM, Ricci S, Mazzaferro V, Hilgard P, Gane E, Blanc JF, et al. Sorafenib in advanced hepatocellular carcinoma. N Engl J Med 2008;359:378-390.

3. Kudo M, Fin RS, Qin S, Han KH, Ikeda K, Piscaglia F, et al. Lenvatinib 
versus sorafenib in first-line treatment of patients with unresectable hepatocellular carcinoma: a randomized phase 3 non-inferiority trial. Lancet 2018;391:1163-1173.

4. Swift B, Nebot N, Lee JK, Han T, Proctor WR, Thakker DR, et al. Sorafenib hepatobiliary disposition: mechanism of hepatic uptake and disposition of generated metabolites. Drug Metab Dispos 2013;41:1179-1186.

5. Hussein Z, Mizuo H, Hayato S, Namiki M, Shumaker R. Clinical pharmacokinetic and pharmacodynamic profile of lenvatinib, an orally active, small module, multitargeted tyrosine kinase inhibitor. Eur J Drug Metab Pharmacokinet 2017;42:903-914.

6. Kaneko S, Furuse J, Kudo M, Ikeda K, Honda M, Nakamoto Y, et al. Guideline on the use of new anticancer drugs for the treatment of Hepatocellular Carcinoma 2010 update. Hepatol Res 2012;42:523542.

7. Heimbach JK, Kulik LM, Finn RS, Sirlin CB, Abecassis MM, Roberts $L R$, et al. AASLD guidelines for the treatment of hepatocellular carcinoma. Hepatology 2018;67:358-380.

8. Song DS, Song MJ, Bae SH, Chung WJ, Jang JY, Kim YS, et al. A comparative study between sorafenib and hepatic arterial infusion chemotherapy for advanced hepatocellular carcinoma with portal vein tumor thrombosis. J Gastroenterol 2015;50:445-454.

9. Oh MJ, Lee HJ, Lee SH. Efficacy and safety of hepatic arterial infusion chemotherapy for advanced hepatocellular carcinoma as firstline therapy. Clin Mol Hepatol 2013;19:288-299.

10. Niizeki T, Sumie S, Torimura T, Kurogi J, Kuromatsu R, Iwamoto $H$, et al. Serum vascular endothelial growth factor as a predictor of response and survival in patients with advanced hepatocellular carcinoma undergoing hepatic arterial infusion chemotherapy. J Gastroenterol 2012;47:686-695.

11. Miyaki D, Aikata H, Honda Y, Naeshiro N, Nakahara T, Tanaka M, et al. Hepatic arterial infusion chemotherapy for advanced hepatocellular carcinoma according to Child-Pugh classification. J Gastroenterol Hepatol 2012;27:1850-1857.

12. Tsai WL, Lai KH, Liang HL, Hsu PI, Chan HH, Chen WC, et al. Hepatic arterial infusion chemotherapy for patients with huge unresectable hepatocellular carcinoma. PLoS One 2014;9:e92784.

13. Terashima T, Yamashita $T$, Arai $K$, Sunagozaka $H$, Kitahara $M$, Nakagawa $\mathrm{H}$, et al. Feasibility and efficacy of hepatic arterial infusion chemotherapy for advanced hepatocellular carcinoma after sorafenib. Hepatol Res 2014;44:1179-1185.

14. Shao YY, Liang PC, Wu YM, Huang CC, Huang KW, Cheng JC, et al. A pilot study of hepatic arterial infusion of chemotherapy for patients with advanced hepatocellular carcinoma who have failed antiangiogenic therapy. Liver Int 2013;33:1413-1419.

15. Kudo M, Izumi N, Kokudo N, Matsui O, Sakamoto M, Nakashima O, et al. Management of hepatocellular carcinoma in Japan: Consensus-Based Clinical Practice Guidelines proposed by the Japan Soci- ety of Hepatology (JSH) 2010 updated version. Dig Dis 2011;29:339364.

16. Kudo M, Matsui $O$, Izumi $N$, lijima $H$, Kadoya $M$, Imai $Y$, et al. JSH consensus-based clinical practice guideline for the management of hepatocellular carcinoma: 2014 update by the Liver Cancer Study Group of Japan. Liver Cancer 2014;3:458-468.

17. Lok AS, Sterling RK, Everhart JE, Wright EC, Hoefs JC, Di Bisceglie $A M$, et al. Des-gamma-carboxy prothrombin and alpha-fetoprotein as biomarkers for the early detection of hepatocellular carcinoma. Gastroenterol 2010;138:493-502.

18. Park SJ, Jang JY, Jeong SW, Cho YK, Lee SH, Kim SG, et al. Usefulness of AFP, AFP-L3, and PIVKA-II, and their combinations in diagnosing hepatocellular carcinoma. Medicine (Baltimore) 2017;96:e5811.

19. Lencioni R, Llovet JM. Modified RECIST (mRECIST) assessment for hepatocellular carcinoma. Semin Liver Dis 2010;30:52-60.

20. National Cancer Institute (NCI). Common Terminology Criteria for Adverse Events. National Institutes of Health web site, <https:// ctep.cancer.gov/protocolDevelopment/electronic_applications/ctc. htm\#ctc_50>. Accessed 15 Jun 2019.

21. Ministry of Health, Labour and Welfare. Ethical Guidelines for Medical and Health Research Involving Human Subjects. Ministry of Health, Labour and Welfare web site, <https://www.mhlw.go.jp/ file/06-Seisakujouhou-10600000-Daijinkanboukouseikagakuka/0000080278.pdf>. Accessed 15 Jun 2019.

22. Lin DY, Liaw YF, Lee TY, Lai CM. Hepatic arterial embolization in patients with unresectable hepatocellular carcinoma--a randomized controlled trial. Gastroenterol 1998;94:453-456.

23. Groupe d'Etude et de Traitement du Carcinome Hépatocellulaire. A comparison of lipiodol chemoembolization and conservative treatment for unresectable hepatocellular carcinoma. N Engl J Med 1995;332:1256-1261.

24. Lo CM, Ngan H, Tso WK, Liu CL, Lam CM, Poon RT, et al. Randomized controlled trial of transarterial lipiodol chemoembolization for unresectable hepatocellular carcinoma. Hepatology 2002;35:11641171.

25. Llovet JM, Real MI, Montaña X, Planas R, Coll S, Aponte J, et al. Arterial embolization or chemoembolization versus symptomatic treatment in patients with unresectable hepatocellular carcinoma: a randomized controlled trial. Lancet 2002;359:1734-1739.

26. Cammà C, Schepis F, Orlando A, Albanese M, Shahied L, Trevisani F, et al. Transarterial chemoembolization for unresectable hepatocellular carcinoma: meta-analysis of randomized controlled trials. Radiology 2002;224:47-54.

27. Llovet JM, Bruix J. Systematic review of randomized trials for unresectable hepatocellular carcinoma: chemoembolization improves survival. Hepatology 2003;37:429-442.

28. Lu W, Li YH, Yu ZJ, He XF, Chen Y, Zhao JB, et al. A comparative 
Kei Moriya, et al.

study of damage to liver function after TACE with use of low-dose versus conventional-dose of anticancer drugs in hepatocellular carcinoma. Hepatogastroenterology 2007;54:1499-1502.

29. Nishikawa H, Osaki Y, Inuzuka T, Takeda H, Nakajima J, Matsuda $F$, et al. Branched-chain amino acid treatment before transcatheter arterial chemoembolization for hepatocellular carcinoma. World J Gastroenterol 2012;18:1379-1384.

30. Ishikawa $T$, Kubota T, Horigome R, Kimura N, Honda H, Iwanaga A, et al. Concurrent partial splenic embolization with transcatheter arterial chemoembolization for hepatocellular carcinoma can maintain hepatic functional reserve. Hepatol Res 2014;44:1056-1061.

31. Pugh RN, Murray-Lyon IM, Dawson JL, Pietroni MC, Williams R. Transection of the oesophagus for bleeding oesophageal varices. $\mathrm{Br}$ J Surg 1973;60:646-649.

32. Montano-Loza AJ. Clinical relevance of sarcopenia in patients with cirrhosis. World J Gastroenterol 2014;20:8061-8071.

33. Moriya K, Namisaki T, Sato S, Douhara A, Furukawa M, Kawaratani $\mathrm{H}$, et al. Efficacy of bi-monthly hepatic arterial infusion chemotherapy for advanced hepatocellular carcinoma. J Gastrointest Oncol 2018:4:741-749.

34. Iwasa S, Ikeda M, Okusaka T, Ueno H, Morizane C, Nakachi K, et al. Transcatheter arterial infusion chemotherapy with a fine-powder formulation of cisplatin for advanced hepatocellular carcinoma refractory to transcatheter arterial chemoembolization. Jpn J Clin
Oncol 2011;41:770-775.

35. Yoshikawa M, Ono N, Yodono H, Ichida T, Nakamura H. Phase Il study of hepatic arterial infusion of a fine-powder formulation of cisplatin for advanced hepatocellular carcinoma. Hepatol Res 2008;38:474-483.

36. Ikeda M, Mitsunaga S, Shimizu S, Ohno I, Takahashi H, Okuyama H,et al. Efficacy of sorafenib in patients with hepatocellular carcinoma refractory to trasncatheter arterial chemoembolization. J Gastroenterol 2014;49:932-940.

37. Hatooka M, Kawaoka T, Aikata H, Morio K, Kobayashi T, Hiramatsu $A$, et al. Comparison of outcome of hepatic arterial infusion chemotherapy and sorafenib in patients with hepatocellular carcinoma refractory to transcatheter arterial chemoembolization. Anticancer Res 2016;36:3523-3529.

38. Kawaoka T, Aikata H, Hyogo H, Morio R, Morio K, Hatooka M, et al. Comparison of hepatic arterial infusion chemotherapy versus sorafenib monotherapy in patients with advanced hepatocellular carcinoma. J Dig Dis 2015;16:505-512.

39. Fukubayashi K, Tanaka M, Izumi K, Watanabe T, Fujie S, Kawasaki T, et al. Evaluation of sorafenib treatment and hepatic arterial infusion chemotherapy for advanced hepatocellular carcinoma: a comparative study using the propensity score matching method. Cancer Med 2015;4:1214-1223. 